\title{
Facile synthesis and characterization of polypyrrole-multiwalled carbon nanotubes by in situ oxidative polymerization
}

\author{
Amin Imani ${ }^{*}$, Gholamali Farzi and Adnen Ltaief
}

\begin{abstract}
Polypyrrole-multiwall carbon nanotube (PPy-MWCNT) nanocomposites were chemically synthesized via in situ oxidative polymerization of pyrrole. Ammonium peroxydisulfate and p-toluenesulfonic acid were used as an initiator and surfactant dopant, respectively. The molar ratio of monomer unit to initiator and dopant was 1:1:1, and the percentage of MWCNT in PPy varied from 1 to $10 \mathrm{wt}$.\%. PPy-MWCNT nanocomposites were characterized to study chemical structure, morphology, thermal, electrical, and surface properties. To accomplish this, the samples have been characterized by Fourier transform infrared spectroscopy, X-ray diffraction, scanning electron microscopy, thermogravimetric analysis, four probe resistivity method, and atomic force microscopy. The results showed that PPy-MWCNT nanocomposites were successfully synthesized via in situ oxidative polymerization method, and also, electrical conductivity of nanocomposites was increased when the content of MWCNT increase.
\end{abstract}

Keywords: Polypyrrole; Multiwall carbon nanotubes; Nanocomposites; Oxidative polymerization; Conductivity; AFM

\section{Background}

It appears that one of the most important group of materials are the conducting polymers with a vast field of applications from primarily passive materials such as coatings and containers to active materials with useful optical, electronic, energy storage, and mechanical properties. They are synthesized either by reduction or oxidation reaction, which is called doping process. Among several conducting polymers, polypyrrole (PPy) has attracted much attention from researchers due to its unique properties such as high conductivity, simple methods of preparation, simply synthesis as a composite matrix, stability, and good mechanical properties. Due to these exceptional properties, it has many potential applications in electronic and electrochromic devices, corrosion protection, rechargeable and lightweight batteries, drug delivery, membrane separation, supercapacitors, and sensors [1-4].

PPy was first synthesized by chemical polymerization during the early nineteenth century [5]. It was found insoluble in most of the common solvents due to the

\footnotetext{
* Correspondence: aminimani@live.com

Department of Materials and Polymer Engineering, Faculty of Engineering, Hakim Sabzevari University, P.O. BOX397, Sabzevar, Iran
}

strong interchain interaction. Usually in chemical polymerization, oxidative agents initiate the chemical reaction. Such a method allows us to categorize oxidative polymerization of pyrrole as a new area in cationic polymerization, in which the conditions of initiation, propagation, and termination of the chains can be said by means of the electrochemical potential of the system. Oxidative polymerization exemplifies a new means to access conducting polymers, and it is one of the important green chemistry processes to prepare multifunctional polymers. This method has been widely used to prepare different types of conducting polymers [6,7] and their different derivatives [8].

PPy can be made chemically through oxidative polymerization of pyrrole. In this neutral state of the PPy, it is not conductive and only becomes conductive when it is oxidized [9]. The charge related with the oxidized state is naturally delocalized over some pyrrole units and can form a radical polaron or a bipolaron [10].

It has also been recognized to be very easy to prepare PPy particles of different sizes ranging from micrometers to nanometers with the addition of different dopants [11]. Nevertheless, PPy provides suitable properties, but to adjust the polymer attributes and attain excellent 
properties, they have been used in composites structure by combining with nanoparticles such as carbon nanotubes $(\mathrm{CNT})$ [12,13]. In general, nanocomposite materials with CNT can show different mechanical, electrical, optical, electrochemical, catalytic, and structural properties than those of each individual component. The multifunctional behavior for any specific property of the material is often more than the sum of the individual components [2]. CNT also have received important concentration for their unique mechanical, electrical, thermal, and magnetic properties that have been used in the field of nanocomposites materials, nanodevices, and so on [4]. The large specific area and aspect ratio of multiwall carbon nanotubes (MWCNTs) have made them efficient as conductive fillers in polymers [14].

In this work, PPy-MWCNT nanocomposites were prepared via in situ chemical oxidative polymerization, and the content of MWCNTs in the composites varied from 1 to $10 \mathrm{wt.} \%$. The impact of different contents of MWCNTs on the structures and properties of the PPy-MWCNT nanocomposites were deeply discussed based on the results from Fourier transform infrared spectroscopy (FT-IR), Xray diffraction (XRD), thermogravimetric analysis (TGA), scanning electron microscopy (SEM), and atomic force microscopy (AFM). Also, the effect of MWCNTs on the electrical properties and electrical temperature dependence of nanocomposites were talked over.

\section{Methods \\ Materials}

Multiwall carbon nanotubes (with 10 to $20 \mathrm{~nm}$ diameter, $30 \mu \mathrm{m}$ length, and density $2.1 \mathrm{~g} / \mathrm{cm} 3$ ) made by chemical vapor deposition were provided by the Petroleum Research Institute of Iran. The purity of the MWCNTs was reported to be $97 \%$. Pyrrole monomer was purchased from Merck, Whitehouse Station, NJ, USA, and purified under low pressure before reaction. Ammonium peroxydisulfate (APS), p-toluenesulfonic acid (p-TSA), and $\mathrm{N}$-methyl-pyrrolidone (NMP) were obtained from Merck and used in our experiment as received. All other chemicals and solvents were of analytical grade and were used without any further purification.

\section{Synthesis of PPy-MWCNT nanocomposites}

The nanocomposites of PPy-MWCNT were synthesized by oxidation polymerization. In a typical procedure, $3.19 \mathrm{~g}$ $(0.014 \mathrm{~mol})$ of APS was dissolved in $75 \mathrm{ml}$ distilled water and stirred for $10 \mathrm{~min}$. A solution of p-TSA in water ( $2.66 \mathrm{~g}$ ( $0.014 \mathrm{~mol})$ of p-TSA in $25 \mathrm{ml}$ water) was added to the aqueous solution of APS and stirred for $15 \mathrm{~min}$. Pyrrole $(1 \mathrm{ml}(0.014 \mathrm{~mol}))$ was mixed to $25 \mathrm{ml}$ water and then $0.0096 \mathrm{~g}$ of MWCNTs was added to the pyrrole solution and ultrasonicated for $10 \mathrm{~min}$. Then, mixture was added slowly to the vessel containing APS/p-TSA solution and stirred about $4 \mathrm{~h}$ at room temperature. The black sediment was washed with ethanol and distilled water consequently until the filtrate was colorless. Then, the specimens were dried under vacuum at $60^{\circ} \mathrm{C}$ for $48 \mathrm{~h}$. Several PPy-MWCNT nanocomposites were synthesized with different weight percent of MWCNTs. For comparison, the pure PPy was also synthesized in the similar manner and used as reference. Also, to study the surface AFM analysis and film formation property of PPy and PPyMWCNT (5\%) nanocomposites in air, several films were prepared according to this procedure: about $0.3 \mathrm{~g}$ of nanocomposites was added to $2 \mathrm{ml}$ of NMP and then stirred for $24 \mathrm{~h}$. Two drops of solution was poured to the prerinsed glass substrate and dried under $40^{\circ} \mathrm{C}$ for $12 \mathrm{~h}$.

\section{Results and discussion}

To characterize the chemical structure of synthesized nanocomposites, the FT-IR was carried out. Figure 1 shows the FT-IR spectra of PPy-MWCNT nanocomposites and pure PPy that was synthesized by in situ chemical oxidative polymerization. The spectra of nanocomposites powder was obtained with $\mathrm{KBr}$ in pellet form. As can be seen in Figure 1, the spectra of nanocomposites are similar to that of the pure PPy. The chief distinctive bands of PPy seen in the spectra of nanocomposites are as follows. The peak at approximately $3,417 \mathrm{~cm}^{-1}$ is attributable to the hydrogenbonded N-H stretching vibration [15]. The peaks at approximately $1,615 \mathrm{~cm}^{-1}$ and approximately $1,547 \mathrm{~cm}^{-1}$ could be attributed to $\mathrm{C}-\mathrm{N}$ and $\mathrm{C}-\mathrm{C}$ asymmetric and symmetric ring stretching, respectively [16,17]. Additionally, the peak near approximately $1,168 \mathrm{~cm}^{-1}$ presents the doping state of PPy $[4,18]$, and the peak at approximately $1,039 \mathrm{~cm}^{-1}$ is attributed to the $\mathrm{C}-\mathrm{H}$ in plane deformation and $\mathrm{N}-\mathrm{H}$ stretching vibration. Inclusion of MWCNT in PPy results in shifting of the peaks. It reveals the interaction of MWCNT with different reaction sites of PPy. The FT-IR results indicate that N-H, $\mathrm{C}-\mathrm{H}$, and $\mathrm{C}-\mathrm{C}$ become weaker, and $\mathrm{C}-\mathrm{N}$ band becomes stronger in the nanocomposites [4] which confirms the formation of C-N bands between PPy and MWCNTs.

TGA of PPy-MWCNT nanocomposites have been performed in the temperature range of $25^{\circ} \mathrm{C}$ to $550^{\circ} \mathrm{C}$. The TGA diagram of PPy-MWCNT nanocomposites and pure PPy were illustrated in Figure 2. As can be seen, there are three steps of weight loss for PPy and PPy-MWCNT samples. The first step of weight loss occurred at $50^{\circ} \mathrm{C}$ to $75^{\circ} \mathrm{C}$ which may be due to the loss of humidity, and about $10 \%$ weight loss occurs. A very small weight loss can be seen around $190^{\circ} \mathrm{C}$ which can be due to the loss of oligomers molecules [19]. The second step of weight loss occurs between $200^{\circ} \mathrm{C}$ and $400^{\circ} \mathrm{C}$ which may be due to the loss of a dopant. The third step of weight loss over the temperature range $400^{\circ} \mathrm{C}$ to $550^{\circ} \mathrm{C}$ 


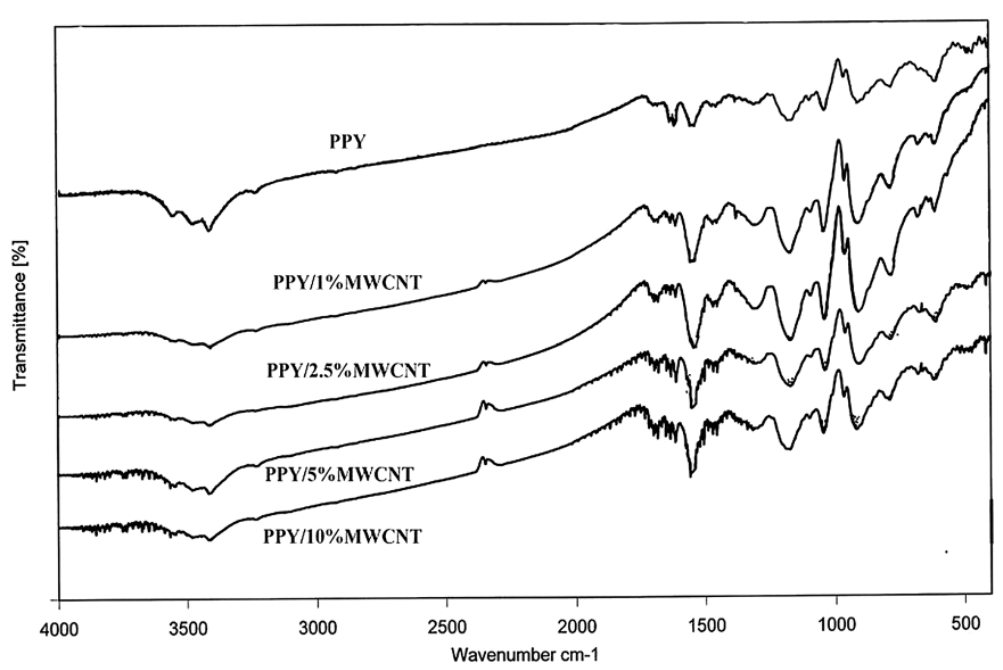

Figure 1 FT-IR spectra of pure PPy and PPy-MWCNT nanocomposites.

is due to the thermal decomposition of PPy into a number of chemical forms. Weight loss that is equal and less than $41 \%$ occurs at $550^{\circ} \mathrm{C}$ for pure PPy and PPy-MWCNT nanocomposites, respectively. Thermal stability of nanocomposites grows partly with increasing MWCNT percentage. However, improvement of thermal stability is not significant due to low weight percent of MWCNTs [4].

The crystallographic arranges of samples are characterized using XRD measurements. Figure 3 shows the XRD pattern of PPy-MWCNT nanocomposites and PPy performed at room temperature. The samples were found to be amorphous in nature that is in accordance with the founding of other researchers $[16,20]$. The XRD pattern of MWCNT is published elsewhere [15], and it shows a sharp, high intensity peak at $2 \theta=$ approximately $26^{\circ}$ and two lower intensity peaks at $2 \theta=$ approximately $45^{\circ}$ and $62^{\circ}$, which are ascribed to the diffraction signature of the distance between the walls and the interwall spacing $[4,15]$. In the case of PPy, peaks appear near $2 \theta=$ approximately $22^{\circ}$ and approximately $26^{\circ}$, which are attributed to the periodicity parallel and perpendicular to the polymer chains $[15,21,22]$. For PPy-MWCNT nanocomposites, the X-ray patterns exhibit both the characteristic peaks of PPy and that of MWCNTs, showing the presence of MWCNT in PPy. The peak at $2 \theta=$ approximately $26^{\circ}$ for PPy-MWCNT nanocomposites is wider

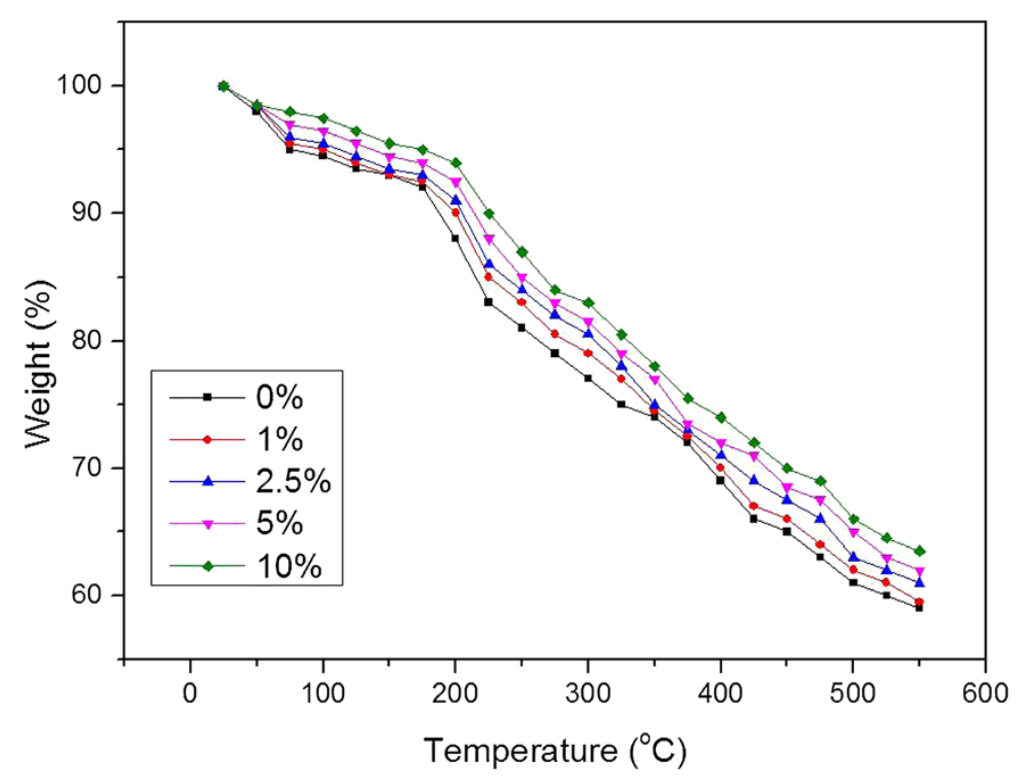

Figure 2 TGA thermograms of PPy and different PPy-MWCNT nanocomposites. 


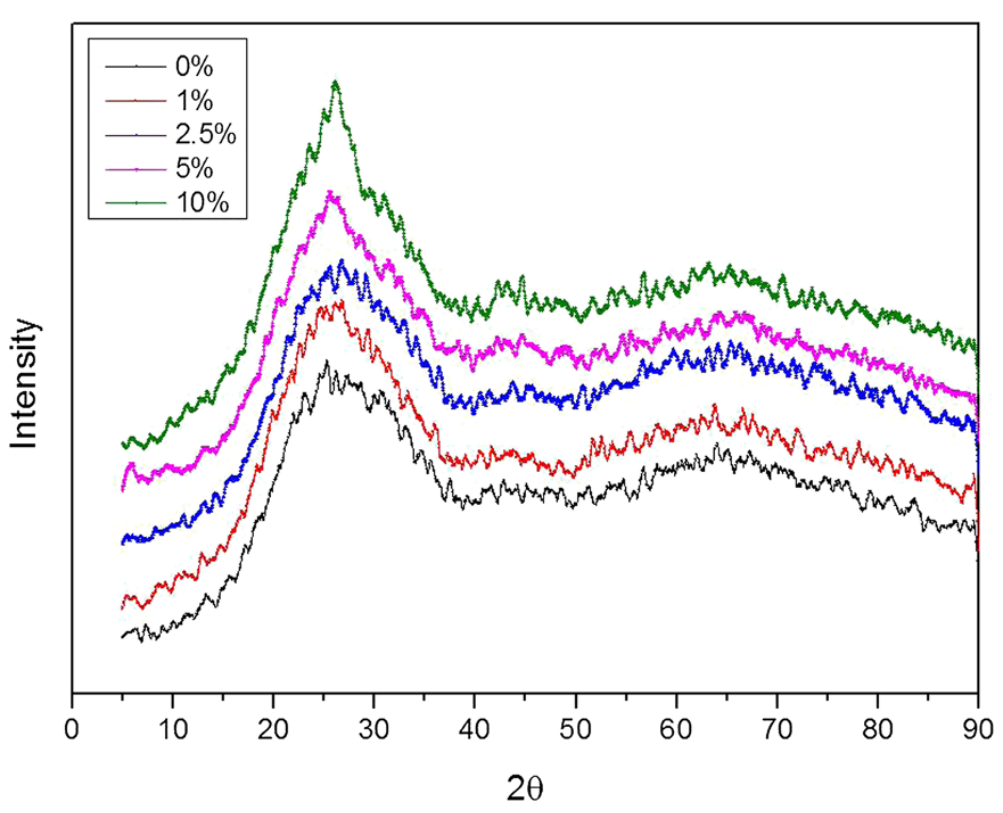

Figure 3 XRD patterns of PPy and PPy-MWCNT nanocomposites.

than that of the pure PPy due to the peak of MWCNT at $2 \theta=$ approximately $26^{\circ}$ that has been merged with the peak of PPy. This indicates that the difference between the patterns of PPy and nanocomposites should be mainly indebted to the stand of the peaks of PPy and MWCNTs [15,18,23,24].

For electrical property studies, PPy and PPy-MWCNT nanocomposites were pressed into cylinder pellet formed under $10 \mathrm{MPa}$ pressure. Then, the conductivity has been measured by a DC voltage/current detector with four probe standard methods at room temperature. The data reported based on the average value of four measurements. The conductivity of nanocomposites has been illustrated vs. MWCNTs weight fraction in Figure 4. As can be seen, the conductivity increases by increasing the MWCNTs content. The structure of the PPy-MWCNT nanocomposites is likely to contribute to the electrical conductivity improvement in the following ways [24]. First, because of the large surface area of MWNCTs, they may act as conducting bridge connecting PPy

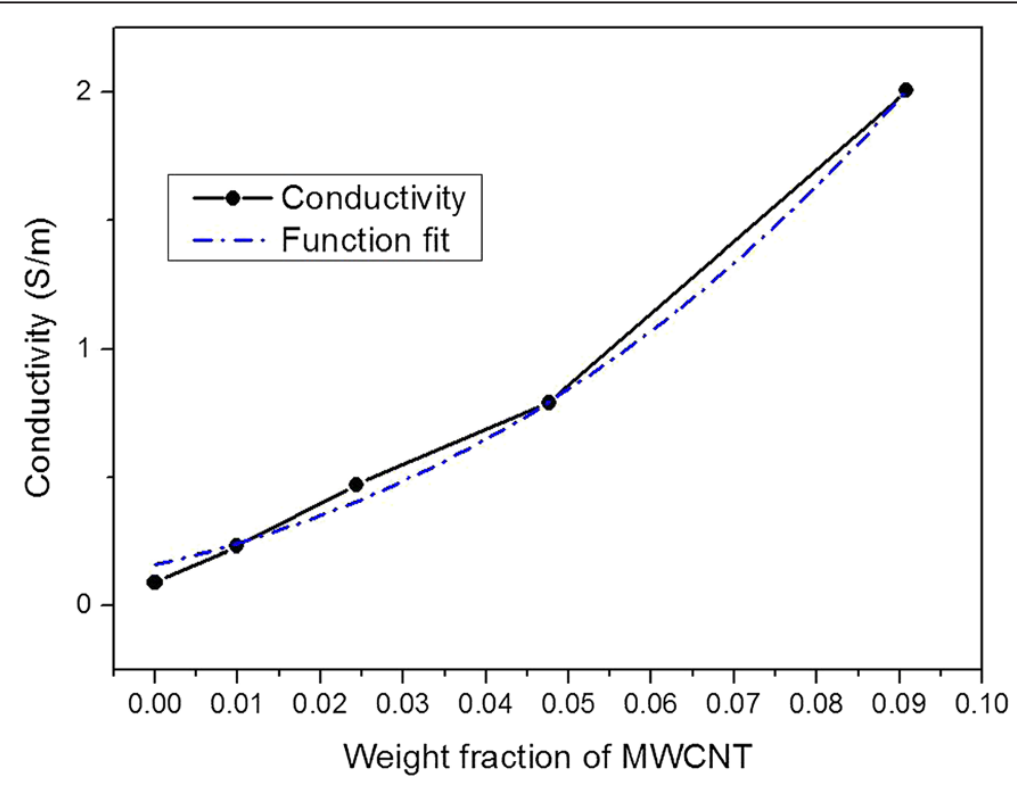

Figure 4 Conductivity plots and percolation threshold function of PPy and PPy-MWCNT nanocomposites. 
Table 1 Values from Figure 4 in percolation threshold equation

\begin{tabular}{lccc}
\hline Equation & $\boldsymbol{\sigma}_{\mathbf{0}}$ & $\boldsymbol{P}_{\mathbf{C}}$ & $\boldsymbol{t}$ \\
\hline$\sigma=\sigma_{0}\left(P-P_{c}\right)^{t}$ & 353.94 & 0.045 & 2.62 \\
\hline
\end{tabular}

conducting domains and increasing the effective penetration [25]. Second, the FT-IR observations suggest that the interaction between PPy and MWCNTs could be responsible for higher conductivity than the starting components $[24,26]$.

In order to gain an understanding to the conductivity percolation threshold, a mathematical function fit was plotted to the experimental values. In mathematics, percolation theory defines the behavior of connected clusters in a random graph [27]. Equation 1 shows the percolation threshold equation:

$$
\sigma=\sigma_{0}\left(P-P_{c}\right)^{t}
$$

where $P$ and $P_{\mathrm{c}}$ are the weight fractions of MWCNTs in the nanocomposite and at the percolation threshold, respectively, $t$ is the percolation exponent (it has a theoretical value of 2 for percolation), and $\sigma_{0}$ is the constant related to the bulk conductivity of MWCNTs (i.e., at $P=1$, $\sigma=\sigma_{\mathrm{MWCNTS}}$ ) [28]. Table 1 indicates the extracted values from conductivity curve. As can be seen clearly, there is a good agreement between experimental and theoretical values $(t=2.62)$. The threshold value for PPy-MWCNT was calculated to be around $4.8 \%$. It seems that the threshold percolation for the conducting matrix (i.e., PPy) in comparison with insulating matrix occurs at higher weight percentage of MWCNTs. It is well known that below the percolation threshold, there is less growth in the conductivity value than above it. For values much greater than percolation threshold, the conductivity was affected to the infinite value [10].

\section{Temperature dependence of conductivity}

Three-dimensional (3D) charge transport mechanism is appropriate for clarifying the temperature dependence of dc conductivity. The temperature dependence of the dc conductivity for conducting polymers tends to follow $3 \mathrm{D}$ variable range hopping (3D VRH) model's prediction (Equation 2) [10,29]:

$$
\sigma=\sigma_{0} \exp \left(-\left(\frac{T_{\text {mott }}}{T}\right)^{\frac{1}{4}}\right)
$$

where $\sigma_{0}$ and $T_{\text {mott }}$ are the conductivity at infinitive temperature and characteristic temperature, respectively. From the 3D VRH model [30]:

$$
T_{\text {mott }}=\frac{18.1}{K_{B} N\left(E_{f}\right) \alpha^{3}}
$$

$K_{\mathrm{B}}$ is Boltzmann's constant $\left(8.616 \times 10^{-5} \mathrm{eV} / \mathrm{K}\right), N\left(E_{F}\right)$ is the density of localized states at the Fermi level $\left(\mathrm{cm}^{-3}\right.$ $\mathrm{eV}^{-1}$ ), and $\alpha$ is the coefficient of exponential decay of the localized length $(\mathrm{cm})$.

To improve the hint about the temperature dependence of dc conductivity of the PPy-MWCNT nanocomposites, the temperature deviation of dc conductivity have been measured contained by a range $80<T<300 \mathrm{~K}$. The values

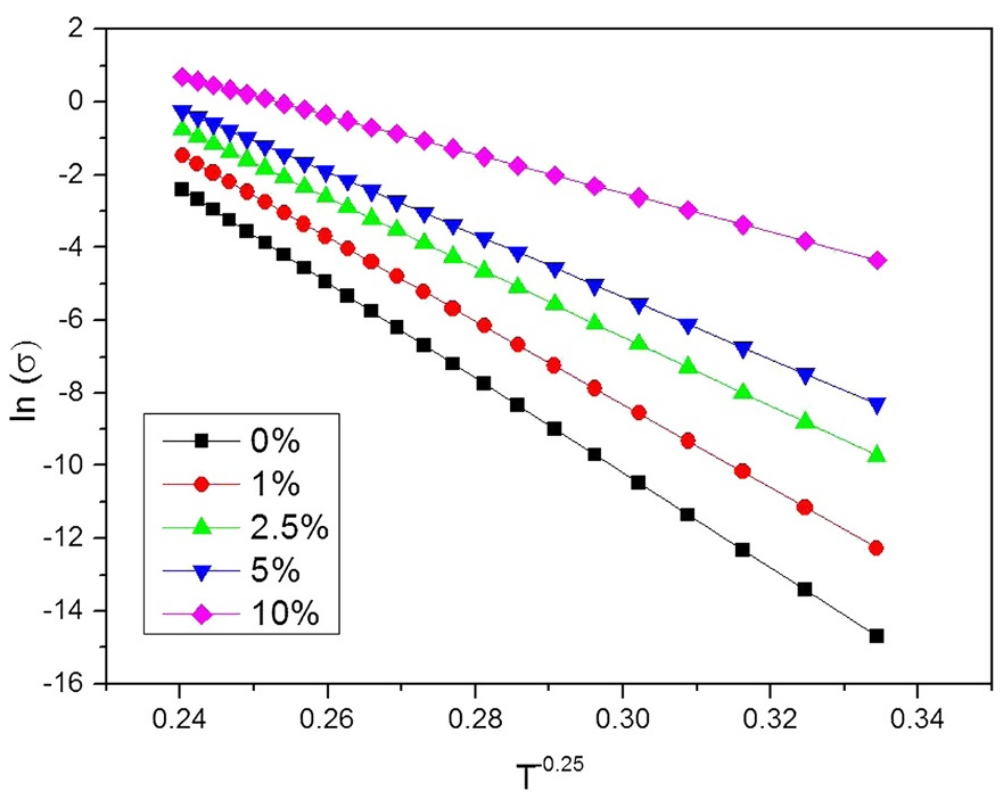

Figure 5 Temperature conductivity dependence of PPy and PPy-MWCNT nanocomposites. 

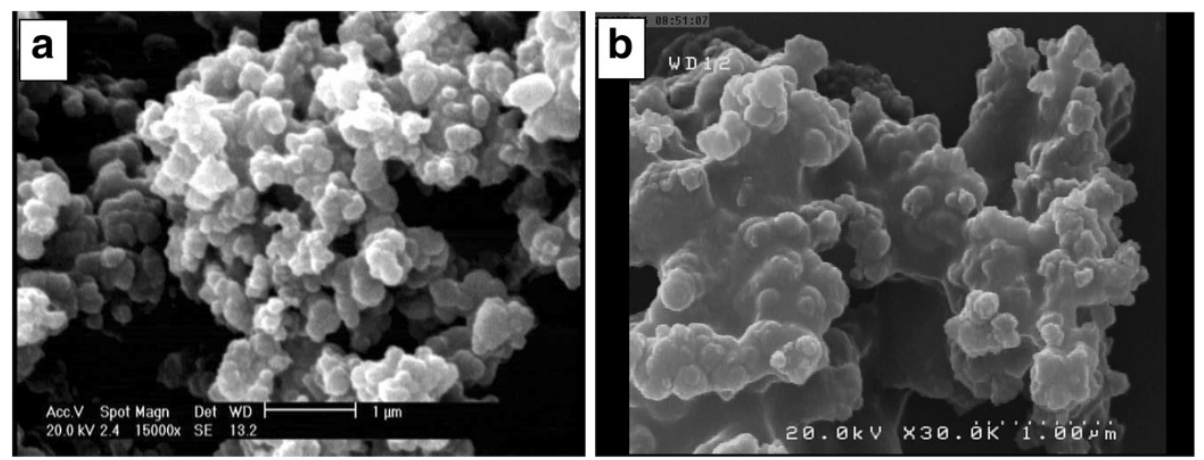

Figure 6 FESEM images of (a) PPy and (b) PPy-MWCNT (10\%).

of conductivity at room temperature have been shown in Figure 4. Resistivity decreases with the combination of MWCNTs in the PPy environment. PPy behaves as a good electron donor though MWCNTs which are somewhat good electron acceptors. Therefore, there are some associations among the quinoid rings of PPy and MWCNTs which facilitate the charge transfer between the two components $[4,17]$. The localization length in MWCNTs is around $10 \mathrm{~nm}$ due to the presence of a large $\pi$-conjugated structure and hence has a high conductivity [4]. However, in the case of PPy, the localization length has been found to be only $1.55 \mathrm{~nm}$ based on our experimental data. Such a small value of localization length results in a relatively poor conductivity. This value is consistent with the values (1 to $2 \mathrm{~nm}$ ) reported by other investigators [10]. Thus, the strong coupling between the MWCNTs and the PPy chains enhances the average localization length and hence conductivity increases for the nanocomposites. Figure 5 shows a plot of $\ln (\sigma)$ vs. $(T)^{-1 / 4}$ in which it could be seen that their dependency is linear.

Figure 6 indicates the FESEM micrograph of PPyMWCNT nanocomposite with 0\% MWCNTs (a) and 10\% MWCNTs (b). As shown by the SEM images, PPy displays a typically cauliflower-like morphology (a) [17,31]. The particle size of PPy was about 150 to $300 \mathrm{~nm}$ with orbicular morphology. This value is consistently reported by other investigators [32]. While XRD configurations specify the existence of MWCNTs in the nanocomposite, the MWCNTs are obscured by means of SEM image (Figure 3), which is comparable to the previous reports for the conventional oxidative polymerized PPy-MWCNTs nanocomposite with $10 \mathrm{wt} . \%$ MWCNTs [33]. The nanocomposite with $10 \%$ MWCNTs (b) indicate both almost spherical collective particles and some unpackaged morphology $[16,34,35]$. Consequently, the FESEM results elucidate the presence of MWCNTs in nanocomposites, and the reactions are remarkably down between MWCNTs and PPy.

AFM imaging of nanocomposite coated glass slides was performed to gain understanding of the topography and roughness of the films. AFM images were mapped using a commercial Solver Next AFM instrument from the NTMDT, Zelenograd, Russia. All mappings were performed in air at room temperature with relative humidity between $20 \%$ and $30 \%$. Figure 7 illustrates $2 \times 2 \mu \mathrm{m}$ AFM images of PPy and PPy-MWCNT (5\%) nanocomposite.

As can be deduced from the scales of the two images, the height of the protuberances detected in pure PPy is
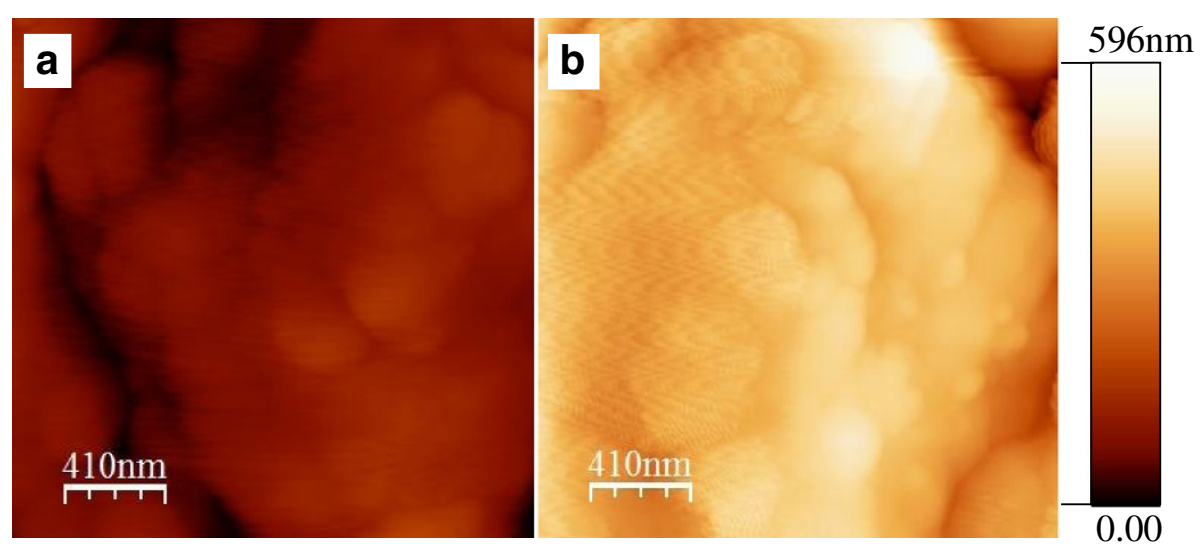

Figure 7 Two AFM topography images of (a) PPy and (b) PPy-MWCNT (5\%). 


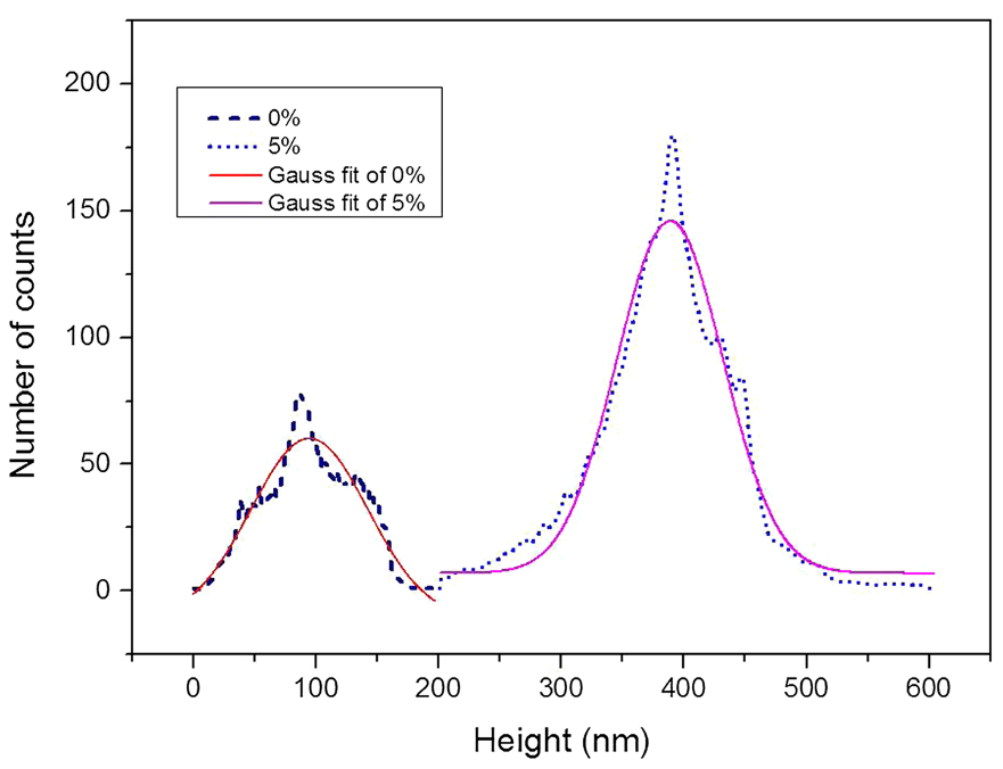

Figure 8 Histograms and simulated normal distribution of the histogram plot corresponding to AFM images.

significantly lower than that obtained for the PPy-MWCNT (5\%). Furthermore, the average roughness determined for PPy and PPy-MWCNT (5\%) films is 54 and $96 \mathrm{~nm}$, respectively.

To rationally understand height values, histogram plots of height images were extracted and normal (Gaussian) distribution of spectrums was attained. Equation 4 shows a single normal (Gaussian) distribution function:

$$
y=\frac{A}{w \sqrt{\frac{\pi}{2}}} e^{-\frac{2\left(x-x_{c}\right)^{2}}{w^{2}}}
$$

where $A$ is a constant number, $y$ symbolizes the number of counts, $w$ is the standard deviation, $x$ represents the height value, and $x_{c}$ is the mean value parameters $[10,36]$. Figure 8 indicates the histogram plots of the number of counts vs. height for PPy-MWCNT (5\%) and PPy. The narrowness of the histograms relevant to MWCNTs containing PPy indicates that the roughness of the surface varies much less than the MWCNT free sample. Evidently, it relates to the presence of MWCNTs in the specimen. However, it can be seen clearly that the number of the pores per unit area is more populated in the case of MWCNTs containing composite. Table 2 shows the extracted normal distribution parameters $\left(w\right.$ and $x_{c}$ ) from height histograms. It can be seen that the standard deviation for PPy-MWCNT is less than the PPy due to the much better interaction of PPy into the MWCNTs surrounding [37]. The increase of the surface roughness is due to the formation of a larger aggregation of MWCNTs as their concentration increases within PPy. The AFM images clearly exhibit a great dependence of the surface topography on the state of MWCNTs incorporation into the polymer matrix.

\section{Conclusions}

In this work, polypyrrole MWCNT nanocomposites have been synthesized successfully by in situ chemical oxidative polymerization method. Structural, morphological, thermal stability, and conductivity studies have been performed for pure PPy and the combination of PPy-MWCNT. The results showed that PPy and PPyMWCNT nanocomposites were successfully synthesized by in situ oxidation polymerization method. The electrical conductivity of nanocomposites increases with increasing wt.\% MWCNTs. The conductivity temperature dependence test showed a strong interaction between the MWCNTs and PPy chains which increases the average localization length so that conductivity increases for the nanocomposite samples. FESEM results confirmed a typically cauliflower-like and orbicular morphology for PPy and PPy-MWCNT nanocomposites. AFM studies indicate a more roughness for PPy-MWCNT (5\%) film than pure PPy and also show interaction between PPy and MWCNTs. More significantly, the oxidative polymerization method seems to be a promoting and effective method that could be readily used for the large scale preparation of the PPy-MWCNT nanocomposites. This method represents a potential application in the production of the polymer-MWCNT nanocomposites.

Table 2 Normal distribution parameters from AFM histogram images

\begin{tabular}{lccc}
\hline Sample & $\boldsymbol{A}$ & $\boldsymbol{w}$ & $\boldsymbol{X}_{\mathbf{c}}$ \\
\hline PPy & $8,445.95$ & 95.45 & 94.75 \\
PPy-MWCNT & $15,144.25$ & 86.92 & 388.82 \\
\hline
\end{tabular}




\section{Abbreviations}

AFM: Atomic force microscopy; APS: Ammonium peroxydisulfate; FT-IR: Fourier transform infrared spectroscopy; MWCNTs: Multiwall carbon nanotubes; PPy: Polypyrrole; p-TSA: p-toluenesulfonic acid; SEM: Scanning electron microscopy; TGA: Thermal analysis gravimeter; VRH: Variable range hopping; XRD: X-ray diffraction.

\section{Competing interests}

The authors declare that they have no competing interests.

\section{Authors' contributions}

FG conceived of the study and participated in its design and coordination. All authors read and approved the final manuscript.

\section{Acknowledgements}

The authors would like to acknowledge the Hakim Sabzevari University for financial support and delivering experimental facilities.

\section{Received: 23 May 2013 Accepted: 31 August 2013} Published: 26 Sep 2013

\section{References}

1. Ansari, R: Polypyrrole conducting electroactive polymers: synthesis and stability studies. E-Journal of Chemistry 3(4), 186-201 (2006)

2. Baytekin, S, Küçükyavuz, Z: Synthesis and characterization of polypyrrole nanoparticles and their nanocomposites with poly(propylene). Macromol. Symp 295, 59-64 (2010)

3. Liu, Y, Chu, Y, Yang, L: Adjusting the inner-structure of polypyrrole nanoparticles through microemulsion polymerization. Mater Chem Phys 98(2-3), 304-308 (2006)

4. Chakraborty, G, Gupta, K, Meikap, AK, Babu, R, Blau, WJ: Synthesis, electrical and magnetotransport properties of polypyrrole-MWCNT nanocomposite. Solid State Commun 152(1), 13-18 (2012)

5. McNeill, R, Siudak, R, Wardlaw, JH, Weiss, DE: Electronic conduction in polymers. I. The chemical structure of polypyrrole. Aust J Chem 16(6), 1056-1075 (1963)

6. Bilici, A, Kaya, I, Yıldırım, M: A comparative study of 9,9-bis(4-aminophenyl) fluorene polymers prepared by catalytic and non-catalytic oxidative polymerisation methods. Eur Polym J 47(5), 1005-1017 (2011)

7. Liao, Y, Li, XG, Kaner, RB: Facile synthesis of water-dispersible conducting polymer nanospheres. ACS Nano 4(9), 5193-202 (2010)

8. $\quad L i, X G$, Huang, MR, Duan, W, Yang, YL: Novel multifunctional polymers from aromatic diamines by oxidative polymerizations. Chem Rev 102(9), 2925-3030 (2002)

9. Li, X, Zhitomirsky, I: Capacitive behaviour of polypyrrole films prepared on stainless steel substrates by electropolymerization. Mater Lett 76, 15-17 (2012)

10. Saville, P: Polypyrrole: Formation and Use. Technical Memorandum, p. 50. DRDC, Atlantic (2005)

11. Selvaraj, M, Palraj, S, Maruthan, K, Rajagopal, G: Polypyrrole as a protective pigment in organic coatings. Synth Met 158(21-24), 888-899 (2008)

12. Li, X, Imin, P, Adronov, A, Zhitomirsky, I: Effect of 5-sulfosalicylic acid and poly[2,5-bis(3-sulfonatopropoxy)-1,4-ethynylphenylene-alt-1,4ethynylphenylene] on electrodeposition of polypyrrole-carbon nanotube films on stainless steel. Mater Lett 68, 24-27 (2012)

13. Rezaul Karim, M, Jae Lee, C, Sarwaruddin Chowdhury, AM, Nahar, N, Sang Lee, M: Radiolytic synthesis of conducting polypyrrole/carbon nanotube composites. Mater Lett 61(8-9), 1688-1692 (2007)

14. Walters, DA, Casavant, MJ, Qin, XC, Huffman, CB, Boul, PJ, Ericson, LM, Haroz, MJ, O'Connell, K, Smith, K, Colbert, DT, Smalley, RE: In-plane-aligned membranes of carbon nanotubes. Chem Phys Lett 338(1), 14-20 (2001)

15. Ubul, A, Abdiryim, T, Jamal, R, Rahman, A: Solid-state synthesis and characterization of polyaniline/multi-walled carbon nanotubes composite. Synth Met 161(19-20), 2097-2102 (2011)

16. Muller, D, Rambo, CR, Porto, LM, Schreiner, WH, Barra, GM: Structure and properties of polypyrrole/bacterial cellulose nanocomposites. Carbohydr Polym 94(1), 655-662 (2013)

17. $\mathrm{Wu}, \mathrm{T}-\mathrm{M}$, Chang, $\mathrm{H}-\mathrm{L}$, Lin, $\mathrm{Y}-\mathrm{W}$ : Synthesis and characterization of conductive polypyrrole/multi-walled carbon nanotubes composites with improved solubility and conductivity. Compos Sci Technol 69(5), 639-644 (2009)
18. Stejskal, J, Sapurina, I, Trchová, M: Polyaniline nanostructures and the role of aniline oligomers in their formation. Prog Polym Sci 35(12), 1420-1481 (2010)

19. Bhadra, S, Kim, NH, Rhee, KY, Lee, JH: Preparation of nanosize polyaniline by solid-state polymerization and determination of crystal structure. Polym Int 58(10), 1173-1180 (2009)

20. Bose, S, Mishra, AK, Kuila, T, Kim, NH, Park, OK, Lee, JH: Tunable electrical conductivity and dielectric properties of triglycine sulfate-polypyrrole composite. Chem Eng J 187, 334-340 (2012)

21. Pintér, E, Patakfalvi, R, Fülei, T, Gingl, Z, Dékany, I, Visy, C: Characterization of polypyrrole-silver nanocomposites prepared in the presence of different dopants. J Phys Chem B 109(37), 17474-17478 (2005)

22. Lu, Y, Pich, A, Adler, HJP, Wang, G, Rais, D, Nespurek, S: Composite polypyrrole-containing particles and electrical properties of thin films prepared therefrom. Polymer 49(23), 5002-5012 (2008)

23. Pande, S, Singh, BP, Mathur, RB, Dhami, TL, Saini, P, Dhawan, SK: Improved electromagnetic interference shielding properties of MWCNT-PMMA composites using layered structures. Nanoscale Res Lett 4(4), 327-334 (2009)

24. Sahoo, NG, Jung, YC, So, HH, Cho, JW: Polypyrrole coated carbon nanotubes: Synthesis, characterization, and enhanced electrical properties. Synth Met 157(8-9), 374-379 (2007)

25. Zengin, H, Zhou, W, Jin, J, Czerw, R, Smith, DW, Echegoyen, L, Carroll, DL, Foulger, L, Ballato, J: Carbon nanotube doped polyaniline. Adv Mater 14(20), 1480-1483 (2002)

26. Wang, $P$, Zheng, Y, Li, B: Preparation and electrochemical performance of poly(3-acetylpyrrole)/multi-walled carbon nanotubes composites. Mater Lett 92, 147-150 (2013)

27. Christensen, K: Percolation Theory, p. 40. Imperial College London, London (2002)

28. Lee, MJ: Pseudo-random-number generators and the square site percolation threshold. Physical Review E 78(3), 031131 (2008)

29. Bellissard, J: A mathematical model for Mott's variable range hopping. Georgia Institute of Technology, Atlanta (2009)

30. Sarmah, S, Kumar, A: Irradiation induced crossover from 1D to 3D transport behaviors of PEDOT-titanium dioxide hybrid nanocomposites. Physica Status Solidi A 209(12), 2546-2551 (2012)

31. Arami, H, Mazloumi, M, Khalifehzadeh, R, Emami, SH, Sadrnezhaad, SK: Polypyrrole/multiwall carbon nanotube nanocomposites electropolymerized on copper substrate. Mater Lett 61(22), 4412-4415 (2007)

32. Chen, S, Zhitomirsky, I: Influence of dopants and carbon nanotubes on polypyrrole electropolymerization and capacitive behavior. Mater Lett 98, 67-70 (2013)

33. Cabezas, AL, Zhang, ZB, Zheng, LR, Zhang, SL: Morphological development of nanofibrillar composites of polyaniline and carbon nanotubes. Synth Met 160(7-8), 664-668 (2010)

34. Amarnath, CA, Ghamouss, F, Schmaltz, B, Autret-Lambert, C, Roger, S, Gervais, F, Tran-Van, F: Polypyrrole/lanthanum strontium manganite oxide nanocomposites: Elaboration and characterization. Synth Met $167,18-24(2013)$

35. Xu, F, Jamal, R, Ubul, A, Shao, W, Abdiryim, T: Characterization and electrochemical properties of poly(aniline-co-o-methoxyaniline)/multi-walled carbon nanotubes composites synthesized by solid-state method. Fibers and Polymers 14(1), 8-15 (2013)

36. Park, SY, Bera, AK: Maximum entropy autoregressive conditional heteroskedasticity model. J Econ 150(2), 219-230 (2009)

37. Chehata, N, Ali Farzi, AL, Ilahi, B, Bouazizi, A: Effect of functionalisation of MWCNTs on optical and morphological properties of MEH-PPV/MWCNTs nanocomposites. Int J Nanotechnol 10, 10 (2013)

\subsection{6/2228-5326-3-52}

Cite this article as: Imani et al: Facile synthesis and characterization of polypyrrole-multiwalled carbon nanotubes by in situ oxidative polymerization. International Nano Letters 2013, 3:52 